\title{
Modeling for Plastic and Reconstructive Breast Surgery
}

\author{
David T. Chen ${ }^{1}$, Ioannis A. Kakadiaris ${ }^{1 \star}$, Michael J. Miller ${ }^{2}$, \\ R. Bowen Loftin ${ }^{1}$, and Charles Patrick ${ }^{2}$
}

1 Virtual Environments Research Institute and Department of Computer Science University of Houston, Houston, TX 77204, USA

\{davechen, ioannisk, bowen\}@uh.edu

2 Laboratory for Reparative Biology \& Bioengineering, Dept. of Plastic Surgery

UT M.D. Anderson Cancer Center Houston, TX 77030, USA

\{mmiller, cpatrick\}@mail.mdanderson.org

\begin{abstract}
In this paper, we present the modeling and estimation aspects of a virtual reality system for plastic and reconstructive breast surgery. Our system has two modes, a model creation mode and a model fitting mode. The model creation mode allows a surgeon to interactively adjust the shape of a virtual breast by varying key shape variables, analogous to the aesthetic and structural elements surgeons inherently vary manually during breast reconstruction. Our contribution is a set of global deformations with very intuitive parameters that a surgeon can apply to a generic geometric primitive in order to model the breast of his/her patient for pre-operative planning purposes and for communicating this plan to the patient. The model fitting mode allows the system to automatically fit a generic deformable model to patient specific three-dimensional breast surface measurements using a physically-based framework. We have tested the accuracy of our technique using both synthetic and real input data with very encouraging results.
\end{abstract}

\section{Introduction}

Virtual reality (VR) has revolutionized many scientific disciplines by providing novel methods to visualize complex data structures and by offering the means to manipulate this data in real-time in a natural way. The most promising fields for the application of VR systems include engineering, education, entertainment, military simulations and medicine. With VR-based systems surgeons are able to: navigate through the anatomy, practice established procedures, practice new procedures, learn how to use new surgical tools, and assess their progress. In particular, the application domain of existing VR applications in surgery can be broadly classified in three categories: a) education and training, b) pre-operative planning, and c) intra-operative assistance. In pre-operative planning the aim is to study patient data before surgery and to plan the best way to carry out that procedure. We are currently developing a VR system that will allow a surgeon to plan and rehearse a plastic and reconstructive breast surgery based on patient specific data.

\footnotetext{
* This work was supported in part by NSF Career Award IIS-9989482.
} 
In this paper, we present the modeling and estimation aspects of a virtual reality system for plastic and reconstructive breast surgery. Our system has two modes, a model creation mode and a model fitting mode. The model creation mode allows a surgeon to interactively adjust the shape of the breast by varying key shape variables, analogous to the aesthetic and structural elements surgeons inherently vary manually during breast reconstruction. Our contribution is a set of global deformations with very intuitive parameters that a doctor can apply to a generic geometric primitive in order to model the breast of his/her patient for pre-operative planning purposes and for communicating this plan to the patient. The model fitting mode allows the system to automatically fit a generic deformable model to patient specific three-dimensional breast surface measurements using a physically-based framework [4,6].

The remainder of this paper is organized as follows. In Section 2 we present the motivation behind our work, and in Section 3 we present the theoretical framework of our research. In particular, in Section 3.1 we describe the basic geometric primitive employed for modeling a breast, in Section 3.2 we formulate the deformations that we have developed, and in Section 3.3 we present the methods employed for fitting a generic virtual breast model to patient-specific breast surface measurements. Finally, in Section 4 we present very encouraging results related to the adequacy of our modeling method and the accuracy of our fitting method using both synthetic and real input data.

\section{Motivation}

Breast size and shape are a significant part of female body image and sense of femininity. Breast deformities can occur due to cancer, congenital and traumatic causes, or the natural changes associated with aging. The psychological impact varies, but some breast deformities are a major cause of morbidity.

Breast deformities. The shape and size of the human female breast is determined by physical characteristics such as tissue volume, skin dimensions, and chest wall circumference. It is a dynamic structure that is soft and easily deformed by position, gravity, and external pressure. The configuration of the mature breast in each individual changes in response to physiologic alterations (e.g., pregnancy, menstrual cycle, etc.) and with advancing age. In addition to these natural changes, deformities can occur as a result of congenital causes, trauma, and cancer treatment.

- Congenital breast deformities: These include both disorders of excessively large breasts (hypermastia), small breasts (hypomastia), and breast asymmetries. Because these disorders generally appear at the time of puberty, they can cause significant emotional problems in teenagers and young adults.

- Postpartum breast deformities: After multiple episodes of breast-feeding, the breast atrophies and becomes ptotic in the late 30's and 40's. This is the most common reason for aesthetic breast surgery to modify the shape and nipple position. 
- Traumatic breast deformities: These are uncommon but include sharp injuries and burns.

- Cancer-related breast deformities: Breast cancer is the most common cancer in women with an incidence of $11 \%$. Deformities related to cancer treatment are the most common type. They range from complete absence of the breast to more limited contour problems resulting from breast conservation treatment (i.e., lumpectomy and radiation). Radiation can cause progressive changes in the breast over many years, making it firmer, rounded, and contracted in areas of scar.

Breast deformities can result in significant emotional and psychological morbidity. As a result, a variety of procedures have been devised to modify the shape and size of the breast.

Plastic surgery for the breast. Plastic surgery is surgery that alters the shape of tissues. Many operations have been devised to alter the female breast. Some enlarge or reduce the size the entire breast. Some alter not the size but only the shape and location of the nipple-areolar complex. Breast reconstruction recreates the entire breast (e.g., after mastectomy) using breast implants or tissue transferred from other parts of the body. Some procedures are a combination of each of these.

Specific techniques for these operations include various skin incisions and methods for removing tissue, adding tissue, inserting prosthetic devises, and fashioning the nipple/areolar complex. It is difficult to predict exactly how the breast will be changed by a specific procedure in any particular patient. How long should incisions be and where should they be placed? What size of breast implant will yield the best results? Is there enough tissue to recreate a breast that would meet the expectations of the patient? What does the patient want her breast to look like? Answering these questions is required for pre-operative planning. There is a certain amount of "trial and error" in the process. The exact result depends heavily on the experience, training, and personal artistic and surgical skills of the individual practitioner. Currently, these procedures are learned by surgeons by operating on actual patients, initially under the instruction of more senior surgeons, then later by independent experience. The patient will not know the final result until after the operation. She must trust the judgment of the surgeon to understand her needs and make many decisions without consulting her. Under these circumstances, the possibility is increased for undesirable outcomes.

Modeling and estimation. The uncertainties associated with breast surgery may be reduced by applying modeling and simulation. A virtual breast simulator may enhance the practice of breast surgery at multiple points. It enables the patient to communicate her expectations more clearly to the surgeon. It allows the surgeon to educate the patient with more accurate explanations of what can be accomplished, and after the patient encounter, it helps the surgeon plan specific aspects of the procedure to achieve the agreed upon goals. Finally, it facilitates surgical training by allowing trainees to design procedures and understand the 
results prior to actually performing surgery on the patient. In this paper, we limit our discussion in the modeling and estimation aspect of our VR system.

\section{Theoretical Framework}

In this section, the theoretical framework that will allow the analysis of a model's deformations is presented. We begin by reviewing the notation for deformable models and then we formulate the global deformations that we developed for modeling the shape of a breast.

\subsection{Deformable Models: Modeling Geometry}

The models used in this work are three-dimensional surface shape models. The material coordinates $\mathbf{u}=(u, v)$ of a point on these models are specified over a domain $\Omega$. The three-dimensional position of a point w.r.t. a world coordinate system is the result of the translation and rotation of its position with respect to a non-inertial, model-centered coordinate frame $\phi$. Therefore, the position of a point (with material coordinates $\mathbf{u}$ ) on a deformable model $i$ at time $t$ with respect to an inertial frame of reference $\Phi$ is given by the formula:

$$
{ }^{\Phi} \mathbf{x}_{i}(\mathbf{u}, t)={ }^{\Phi} \mathbf{t}_{i}(t)+{ }_{\phi}^{\Phi} \mathbf{R}_{i}(t){ }^{\phi} \mathbf{p}_{i}(\mathbf{u}, t),
$$

where ${ }^{\Phi} \mathbf{t}_{i}$ is the position of the origin $O_{i}$ of the model frame $\phi_{i}$ with respect to the frame $\Phi$ (the model's translation), and $\underset{\phi_{i}}{\Phi} \mathbf{R}_{i}$ is the matrix that encapsulates the orientation of $\phi_{i}$ with respect to $\Phi[4,7] .{ }^{\phi_{i}} \mathbf{p}(\mathbf{u}, t)$ is the position of a model point with material coordinates $\mathbf{u}$ w.r.t. the model frame $i$ and can be expressed as the sum of a reference shape ${ }^{\phi_{i}} \mathbf{S}(\mathbf{u}, t)$ and a local displacement ${ }^{\phi_{i}} \mathbf{d}(\mathbf{u}, t)$ as given by the formula: ${ }^{\phi_{i}} \mathbf{p}(\mathbf{u}, t)={ }^{\phi_{i}} \mathbf{s}(\mathbf{u}, t)+{ }^{\phi_{i}} \mathbf{d}(\mathbf{u}, t)$. The reference shape captures the salient shape features of the model and it is the result of applying global deformations $\mathbf{T}$ to a geometric primitive $\mathbf{e}=$ $\left[e_{x}, e_{y}, e_{z}\right]^{\top}$. The geometric primitive $\mathbf{e}$ is defined parametrically in $\mathbf{u} \in \Omega$ and has global shape parameters $\mathbf{q}$. For the purposes of this research, we employ a superquadric $\mathbf{e}(u, v):\left[-\frac{\pi}{2}, \frac{\pi}{2}\right) x[-\pi, \pi) \rightarrow \mathbb{R}^{3}$, whose global shape parameters are $\mathbf{q} \mathbf{e}=\left[a_{1}, a_{2}, a_{3}, \epsilon_{1}, \epsilon_{2}\right]^{\top^{2}}$. A superquadric surface is defined by a vector sweeping a closed surface in space by varying the material coordinates $u$ and $v$. The parametric equation of a superquadric is given by the formula $[2,1]$ :

$$
\mathbf{e}(\mathbf{u})=\left[a_{1} C_{u}{ }^{\epsilon_{1}} C_{v}{ }^{\epsilon_{2}}, a_{2} C_{u}{ }^{\epsilon_{1}} S_{v}{ }^{\epsilon_{2}}, a_{3} S_{u}{ }^{\epsilon_{1}}\right]^{\top},
$$

where $-\frac{\pi}{2} \leq u \leq \frac{\pi}{2},-\pi \leq v \leq \pi, a_{1}, a_{2}, a_{3} \geq 0$ are the parameters that define the superquadric size, and $\epsilon_{1}$ and $\epsilon_{2}$ are the "squareness" parameters in the latitude and longitude plane, respectively. To model the shape of a breast, we only need half of the $(u, v)$ space, therefore in our case $0 \leq u \leq \frac{\pi}{2}$. In addition, in order to be able to vary the sizes of different halves of the superquadric, we employ an asymmetric superquadric with the following parameters: $\mathbf{q}_{\mathbf{e}}=\left[a_{1 \mathrm{~b}}, a_{1 \mathrm{t}}, a_{2 \mathrm{l}}, a_{2 \mathrm{r}}, a_{3}, \epsilon_{1}, \epsilon_{2}\right]^{\top}$, where $a_{1 \mathrm{~b}}$ relates to the bottom half of 

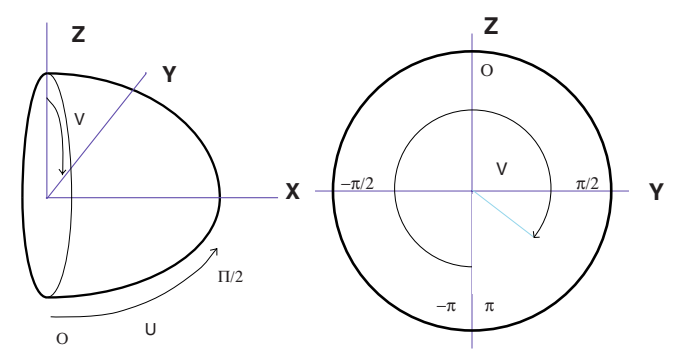

Fig. 1. Side and front views of the virtual breast model and of its associated coordinate systems.

the superquadric $\left(|v| \geq \frac{\pi}{2}\right), a_{1 t}$ relates to the top half $\left(|v|<\frac{\pi}{2}\right), a_{2 l}$ corresponds to the left half $(v \geq 0)$, and $a_{2 r}$ to the right half $(v<0)$.

The local coordinate system for our asymmetric superquadric has the $x$ axis protruding outward through the nipple, the $z$ axis going up, and the $y$ axis goes to the patient's life (Fig. 1). The deformations described in this paper are all expressed in this coordinate system.

\subsection{Global Deformations}

In our system, we model (using global deformations) five major features of the shape of the breast. First, the Ptosis deformation models the sagging that affects a breast as a subject ages. Second, the Turn deformation causes the breast to point towards the left or the right. Third, the Top-Shape deformation models the concavity/convexity of the profile of the top half of the breast. For an ideal breast shape the top half is concave, however in cases where large implants have been inserted into the breast, the top half becomes convex. Fourth, the FlattenSide deformation flattens the shape of the half of the breast towards the middle of the torso. Finally, the Turn-Top deformation turns the top half of the breast towards the shoulder. In particular, the shape of the virtual breast model is given by ${ }^{\phi_{i}} \mathbf{s}(\mathbf{u}, t)=\left[s_{x}, s_{y}, s_{z}\right]^{\top}=\mathbf{T}\left(\mathbf{e} ; \mathbf{q}_{\mathrm{T}}\right)$, where the global deformations $\mathbf{T}$ depend on the parameters $\mathbf{q}_{\mathrm{T}}$. In the following, we provide in detail the global deformations employed for modeling the breast.

Ptosis deformation. The Ptosis deformation depends on the parameters $\mathbf{q}_{\mathrm{T}}=\left[b_{0}, b_{1}\right]^{\top}$ and it is modeled as a quadratic function with coefficients $b_{0}$ and $b_{1}$. The deformation affects a point's vertical position $(z)$ as a function of its depth $(x)$. In particular, the Ptosis deformation $\mathbf{s}=\mathbf{T}_{p}\left(\mathbf{e} ; b_{0}, b_{1}\right)$ along a centerline parallel to the $z$-axis of a primitive $\mathbf{e}=\left[e_{x}, e_{y}, e_{z}\right]^{\top}$ is given by:

$$
s_{x}=e_{x}, s_{y}=e_{y}, s_{z}=e_{z}-\left(b_{0} e_{x}+b_{1} e_{x}^{2}\right) .
$$

Figure 2 depicts front and side views of a deformable breast model to which Ptosis deformation has been applied.

Turn deformation. The Turn deformation causes the shape of the breast to turn to the left or to the right. It uses a quadratic function to scale the $y$ 

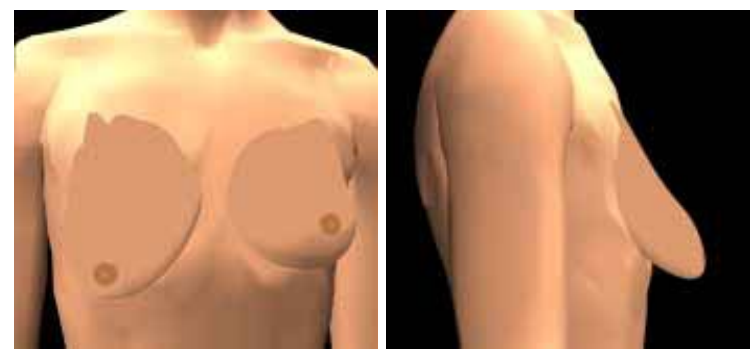

Fig. 2. Examples of Ptosis deformation with parameters $\left(b_{0}, b_{1}\right)=(0.479,-0.160)$ for the virtual patient's left breast, and $\left(b_{0}, b_{1}\right)=(0.691,0.479)$ for the right breast.

coordinate of a model point as a function of its $x$ coordinate. The parameters of the deformation are $\mathbf{q}_{\mathrm{T}}=\left[c_{0}, c_{1}\right]^{\top}$, where $c_{0}$ controls the first order turning rate, and $c_{1}$ controls the second order turning. In particular, the Turn deformation $\mathbf{s}=\mathbf{T}_{c}\left(\mathbf{e} ; c_{0}, c_{1}\right)$ along a centerline parallel to the $y$-axis of a primitive $\mathbf{e}$ is given by:

$$
s_{x}=e_{x}, s_{y}=e_{y}\left(c_{0} e_{x}+c_{1} e_{x}^{2}\right), s_{z}=e_{z} .
$$

Figure 3 depicts front and top views of a virtual patient's right breast to which turn deformation has been applied.

Top-Shape deformation. The Top-Shape deformation allows the user to modify the shape of the top half of the breast $\left(-\frac{\pi}{2} \leq v \leq \frac{\pi}{2}\right)$. For such a point, we apply a polynomial that scales its $z$ coordinate as a function of its $u$ value (i.e., the point's longitude where the nipple is the north pole). In addition, $u^{\prime}=u \frac{2}{\pi}$ and spans the range $[0,1]$. The deformation's parameters are $\mathbf{q}_{\mathrm{T}}=\left[s_{0}, t_{0}, s_{1}, t_{1}\right]^{\top}$, where $s_{0}$ is the slope for $u^{\prime}=0, t_{0}$ is the curvature for $u^{\prime}=0, s_{1}$ is the slope for $u^{\prime}=1$, and $t_{1}$ is the curvature for $u^{\prime}=1$. The slope parameters allow the user to vary the slope of the points near the torso and near the nipple. Similarly, the curvature parameters allow the user to adjust the curvatures near the torso and near the nipple. The Top-Shape deformation $\mathbf{s}=\mathbf{T}_{s}\left(\mathbf{e} ; s_{0}, t_{0}, s_{1}, t_{1}\right)$ is formulated as:

$$
s_{x}=e_{x}, s_{y}=e_{y}, s_{z}=e_{z} \mathbf{f}_{1}\left(u^{\prime}\right)
$$
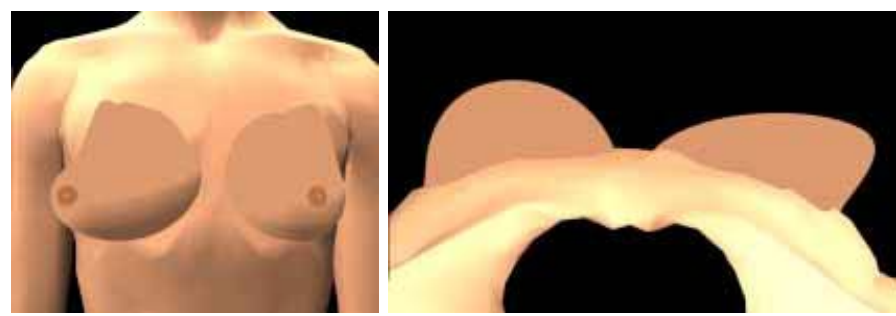

Fig. 3. Examples of Turn deformation with parameters $\left(c_{0}, c_{1}\right)=(-0.053,0)$ for the virtual patient's left breast, and $\left(c_{0}, c_{1}\right)=(0.798,0.213)$ for the patient's right breast. 


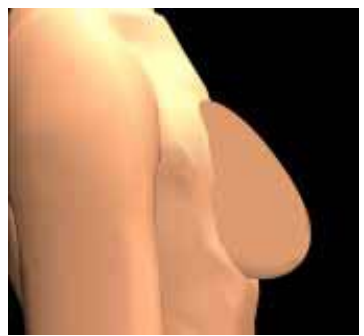

(a)

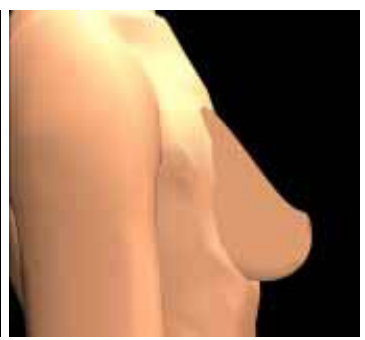

(b)

Fig. 4. Examples of Top-Shape deformation with parameters $\left(s_{0}, t_{0}, s_{1}, t_{1}\right)=$ $(.904,-7.979,0,0)$ (left image) and $\left(s_{0}, t_{0}, s_{1}, t_{1}\right)=(-1.543,-6.915,2.287,-1.064)$ (right image).

In determining the order of the polynomial and its coefficients, we seek to adhere to the following constraints. The positions of the points where the breast merges with the torso and of the nipple must stay fixed, thus $\mathbf{f}_{1}(0)=1$ and $\mathbf{f}_{1}(1)=1$. The following equalities must hold for the slope parameters: $\mathbf{f}_{1}^{\prime}(0)=$ $s_{0}$ and $\mathbf{f}_{1}^{\prime}(1)=s_{1}$. Finally, curvature parameters should satisfy the following equalities: $\mathbf{f}_{1}^{\prime \prime}(0)=t_{0}$ and $\mathbf{f}_{1}^{\prime \prime}(1)=t_{1}$. With this six constraints our polynomial is a quintic with coefficients $A$ through $F$ as follows:

$$
\mathbf{f}_{1}\left(u^{\prime}\right)=A u^{\prime 5}+B u^{\prime 4}+C u^{\prime 3}+D u^{\prime 2}+E u^{\prime}+F
$$

where $A=-\frac{1}{2} t_{0}-3 s_{0}-3 s_{1}+\frac{1}{2} t_{1}, B=\frac{3}{2} t_{0}+8 s_{0}+7 s_{1}-\frac{1}{2} t_{1}, C=-\frac{3}{2} t_{0}-6 s_{0}-$ $4 s_{1}+\frac{1}{2} t_{1}, D=\frac{1}{2} t_{0}, E=s_{0}$, and $F=1$. Figure 4 depicts side views of examples of the Top-Shape deformation applied to the right breast. In Fig.4(a) the slope at the torso is positive, creating a convex upper breast, while in $4(\mathrm{~b})$ the slope at the nipple is negative, creating a dip near the nipple.

Flatten-Side deformation. The shape of the breast has a tendency to flatten out as it approaches the sternum. To achieve this shape, we use the Flatten-Side deformation that affects the inner half of the breast. For example, for a patient's left breast the deformation flattens the breast's right side and similarly for a patient's right breast the deformation flattens the breast's left side (as seen by the patient's point of view). The right side of the virtual breast model includes points for which $v \leq 0$, while the left side includes points for which $v>0$.

This deformation uses a cubic function that scales a point's $x$ coordinate as a function of its horizontal position $(y)$. The cubic is given by:

$$
\mathbf{f}_{2}\left(y^{\prime}\right)=A y^{\prime 3}+B y^{\prime 2}+C y^{\prime}+D,
$$

where $y^{\prime}$ is normalized to $[0,1]$ from the middle of the breast to the sternum. The parameters of the deformation are $\mathbf{q}_{\mathrm{T}}=\left[g_{0}, g_{1}\right]^{\top}$, where $g_{0}$ controls the scaling of points towards the sternum, and $g_{1}$ controls the change in the scaling as points move towards the middle of the breast. Since the function should not affect the breast at the nipple, we apply the constraint $\mathbf{f}_{2}(0)=1$ and $\mathbf{f}_{2}^{\prime}(0)=0$. For 


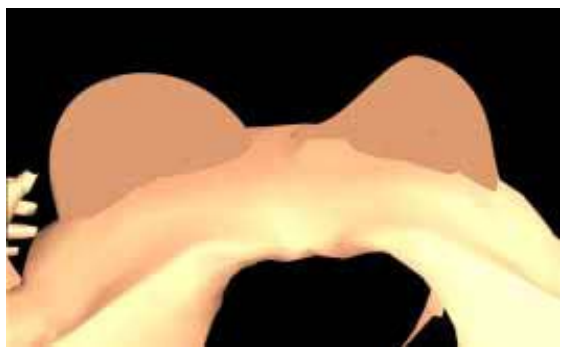

(a)

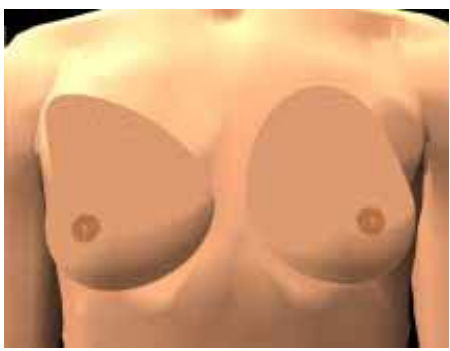

(b)

Fig. 5. (a) Example of a Flatten-Side deformation with parameters $\left(g_{0}, g_{1}\right)=(1,0)$. (b) Example of a Turn-Top deformation with parameters $\left(h_{0}, h_{1}\right)=(0.266,0.372)$ for the virtual patient's right breast.

our parameters $\mathbf{f}_{2}(1)=g_{0}$ and $\mathbf{f}_{2}^{\prime}(1)=g_{1}$. Thus, the Flatten-Side deformation $\mathbf{s}=\mathbf{T}_{g}\left(\mathbf{e} ; g_{0}, g_{1}\right)$ is given by:

$$
s_{x}=e_{x}, s_{y}=e_{y} \mathbf{f}_{2}\left(e_{y^{\prime}}\right), s_{z}=e_{z},
$$

where $\mathbf{f}_{2}\left(y^{\prime}\right)=A y^{\prime 3}+B y^{\prime 2}+C y^{\prime}+D, A=g_{1}+2-2 g_{0}, B=-g_{1}-3+3 g_{0}, C=0$, and $D=1$. Figure $5(\mathrm{a})$ depicts a top-down view of a Flatten-Side deformation applied to a virtual patient's right breast.

Turn-Top deformation. The Turn-Top deformation changes the shape of the top half of the breast laterally. This deformation allows the top part of the virtual breast to point towards the shoulder. The deformation uses a quadratic function to adjust a point's $y$ coordinate as a function of its normalized $z$ coordinate, $z^{\prime}$. Its parameters are $\mathbf{q}_{\mathrm{T}}=\left[h_{0}, h_{1}\right]^{\top}$, and it is applied to points for which $-\frac{\pi}{2} \leq v \leq \frac{\pi}{2}$. In particular, the Turn Top deformation $\mathbf{s}=\mathbf{T}_{h}\left(\mathbf{e} ; h_{0}, h_{1}\right)$ along a centerline parallel to the $y$-axis of a primitive $\mathbf{e}$ is given by:

$$
s_{x}=e_{x}, s_{y}=e_{y}-h_{0} e_{z}-h_{1} e_{z^{\prime}}^{2}, s_{z}=e_{z} .
$$

Figure 5(b) depicts a Turn-Top deformation applied to a virtual patient's right breast.

\subsection{Fitting}

Through the application of Lagrangian mechanics, the geometric parameters of the virtual breast deformable model, the global (parameterized) and local (freeform) deformation parameters, and the six degrees of freedom of rigid-body motion are systematically converted into generalized coordinates or dynamic degrees of freedom [7,5]. The resulting Lagrangian equations are of the form $\dot{\mathbf{q}}+\mathbf{K q}=\mathbf{f}_{q}$, for shape estimation tasks, where $\mathbf{K}$ is the stiffness matrix, $\mathbf{f}_{q}$ are the generalized external forces that act on the model, and $\mathbf{q}$ are the model's generalized coordinates. The damping and the stiffness matrices determine the viscoelastic properties of the deformable model. The elastic properties of the 
Table 1. The parameters for the virtual breast model depicted in Fig. 6(b)

\begin{tabular}{lll}
\hline Deformation & Left Breast & $\begin{array}{c}\text { Parameter Values } \\
\text { Right Breast }\end{array}$ \\
\hline Top-Shape & $(-0.532,-7.447,1.277,0.000)$ & $(-0.532,-6.383,1.702,0.000)$ \\
Ptosis & $(1.0110,0.000)$ & $(1.0110,0.000)$ \\
Turn & $(0.106,0.000)$ & $(0.000,0.000)$ \\
Flatten-Side & $(1.000,0.000)$ & $(1.000,0.000)$ \\
Turn-Top & $(-0.160,-1.489)$ & $(0.160,1.489)$ \\
\hline
\end{tabular}

Table 2. The parameters for the virtual breast model depicted in Fig. 6(d)

\begin{tabular}{lll}
\hline Deformation & Left Breast & $\begin{array}{c}\text { Parameter Values } \\
\text { Right Breast }\end{array}$ \\
\hline Top-Shape & $(-2.234,8.511,1.064,-0.532)$ & $(-2.234,8.511,1.064,-0.532)$ \\
Ptosis & $(0.798,0.851)$ & $(0.638,0.745)$ \\
Turn & $(0.000,-0.053)$ & $(0.213,-0.053)$ \\
Flatten-Side & $(0.505,0.000)$ & $(0.452,0.000)$ \\
Turn-Top & $(-0.106,-0.638)$ & $(0.319,0.638)$ \\
\hline
\end{tabular}

virtual breast model are being adapted in space and in time using the techniques described in $[4,6]$. In physics-based shape estimation techniques, data points apply forces to the deformable model. These forces that the data apply to the model are converted to generalized 3D forces. Based on these forces the model will deform to minimize the discrepancy between the model and the data.

\section{Experimental Results}

In order to access the adequacy of the proposed global transformations, we have performed a number of experiments where a plastic surgeon constructs a virtual deformable model for a breast depicted in an image. The example images depicted in Figs. 6(a,c,e) have been randomly selected from [3]. Figures 6(b,d,f) depict the deformable models build by the surgeon. The parameters for these virtual breast models are detailed in Tables 1, 2, and 3, respectively.

Furthermore, in order to access the accuracy of the fitting, we have performed a number of shape estimation experiments with both synthetic and real data. Figure 6(g) depicts range data obtained from a subject using a Cyberware scanner, while Fig. 6(h) depicts the estimated model. The parameters for the estimated deformable breast model are detailed in Table 4.

\section{Conclusion}

In this paper, we have presented the modeling and shape estimation module of a VR system for plastic and reconstructive breast surgery. In particular, we presented the global deformations that enables us to model a female breast. We have 
Table 3. The parameters for the virtual breast model depicted in Fig. 6(f)

\begin{tabular}{lll}
\hline Deformation & Left Breast & $\begin{array}{c}\text { Parameter Values } \\
\text { Right Breast }\end{array}$ \\
\hline Top-Shape & $(-1.064,7.979,0.532,0.000)$ & $(-1.064,7.979,0.532,0.000)$ \\
Ptosis & $(0.319,0.000)$ & $(0.266,0.106)$ \\
Turn & $(0.266,-0.372)$ & $(-0.053,0.319)$ \\
Flatten-Side & $(1.000,0.000)$ & $(1.000,0.000)$ \\
Turn-Top & $(0.319,0.638)$ & $(0,0)$ \\
\hline
\end{tabular}

Table 4. Parameter values for the estimated deformable model (Fig. 6(h))

\begin{tabular}{ll}
\hline Deformation & Parameter Values \\
\hline Top-Shape & $(-1.543,-6.915,1.915,-2.128)$ \\
Ptosis & $(0.213,-0.160)$ \\
Turn & $(0.160,0.000)$ \\
Flatten-Side & $(0.319,-1.489)$ \\
Turn-Top & $(0.160,-0.372)$ \\
\hline
\end{tabular}

presented several modeling examples along with very encouraging results from fitting a generic deformable breast model to three-dimensional data obtained using range scanning techniques.

\section{References}

1. A. Barr. Global and local deformations of solid primitives. Computer Graphics, 18(3):21-30, 1984.

2. A. H. Barr. Superquadrics and angle-preserving transformations. IEEE Computer Graphics and Applications, 1(1):11-23, January 1981.

3. John Bostwick III. Plastic and Reconstructive Breast Surgery. Quality Medical Publishing, St. Louis, Missouri, 1990.

4. I. A. Kakadiaris. Motion-Based Part Segmentation, Shape and Motion Estimation of Multi-Part Objects: Application to Human Body Tracking. PhD dissertation, Dept of Computer and Information Science, Univ. of Pennsylvania, Philadelphia, PA, Oct. 1996.

5. I. A. Kakadiaris and D. Metaxas. 3D Human body model acquisition from multiple views. International Journal on Computer Vision, 30(3):191-218, 1998.

6. D. Metaxas and I. A. Kakadiaris. Elastically adaptive deformable models. In Bernard Buxton and Roberto Cipolla, editors, Proceedings of the Fourth European Conference on Computer Vision, Lecture Notes in Computer Science, pages II:550 559, Cambridge, UK, April 14-18 1996.

7. D. Metaxas and D. Terzopoulos. Shape and nonrigid motion estimation through physics-based synthesis. IEEE Transactions on Pattern Analysis and Machine Intelligence, 15(6):580-591, June 1993. 


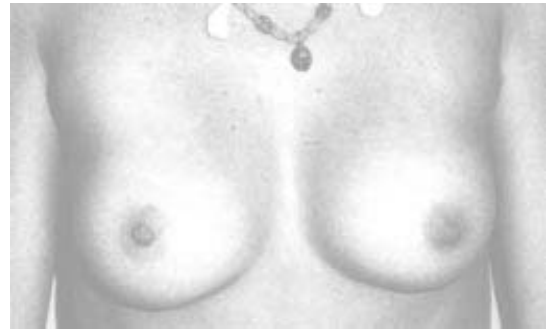

(a)

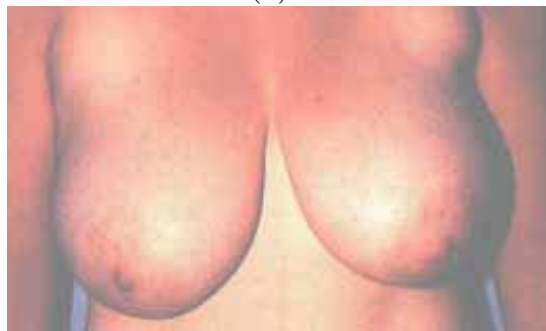

(c)

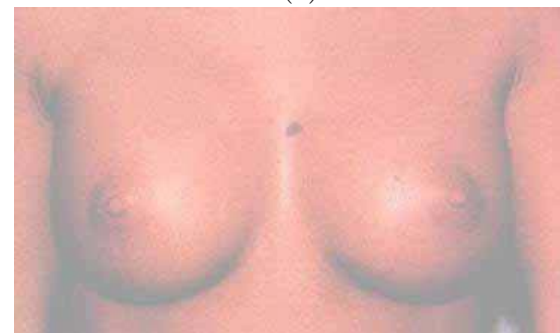

(e)

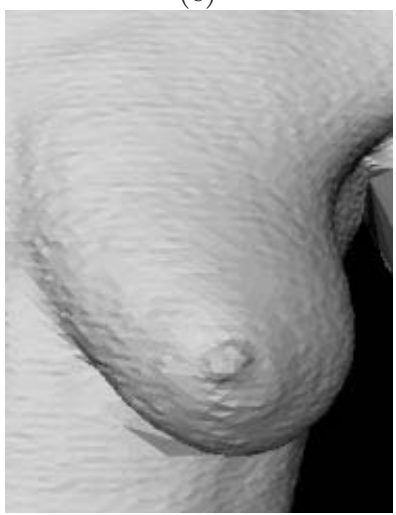

$(\mathrm{g})$

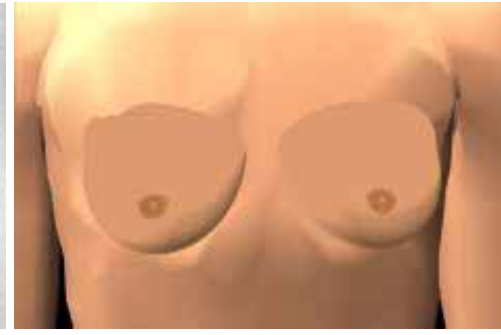

(b)

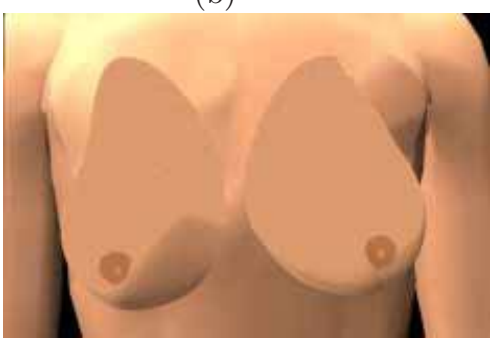

(d)

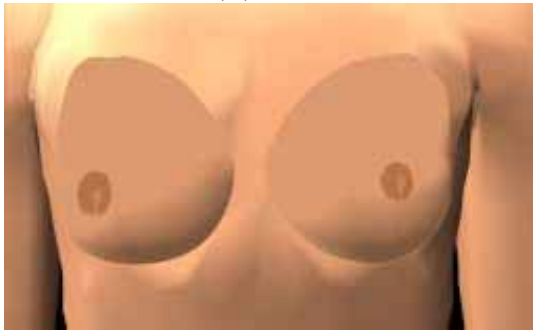

(f)

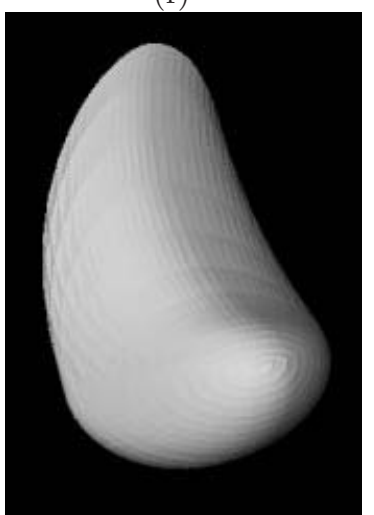

(h)

Fig. 6. (b,d,f) Examples of virtual breast models developed by a surgeon to model the female breasts depicted in $(\mathrm{a}, \mathrm{c}, \mathrm{e}) .(\mathrm{g}, \mathrm{h})$ Range data from a patient's breast and the estimated deformable model. 